\title{
Efficacy of moxifloxacin in the treatment of bronchial colonisation in COPD
}

\author{
M. Miravitlles*, A. Marín\#, E. Monsó ", S. Vilà*, C. de la Roza+, R. Hervás๘, \\ C. Esquinas*, M. García", L. Millares ${ }^{\star}$, J. Morera" and A. Torres ${ }^{\S}$
}

ABSTRACT: This study was designed to investigate the efficacy of moxifloxacin for the eradication of bacterial colonisation of the airways in patients with moderate-to-severe chronic obstructive pulmonary disease (COPD).

Out of 119 stable patients with COPD screened, 40 (mean age 69 yrs, mean forced expiratory volume in 1 s $50 \%$ predicted) were colonised with potentially pathogenic microorganisms (PPMs) and were included in a randomised, double-blind, placebo-controlled trial with moxifloxacin $400 \mathrm{mg}$ daily for 5 days.

Eradication rates were $75 \%$ with moxifloxacin and $30 \%$ with placebo at 2 weeks $(p=0.01)$. Bacterial persistence at 8 weeks was still higher (not significantly) in the placebo arm (five (25\%) out of 20 versus one (5\%) out of $20 ; p=0.18$ ). The frequencies of acquisition of a new PPM were high and similar in both treatment groups; consequently, the prevalence of colonisation at 8 weeks was also similar between treatment arms. No difference was found in the number of patients with exacerbations during the 5-month follow-up. Only the acquisition of a new PPM during follow-up showed a statistically significant relationship with occurrence of an exacerbation.

Moxifloxacin was effective in eradicating PPMs in patients with positive sputum cultures. However, most patients were recolonised after 8 weeks of follow-up. Acquisition of a new strain of bacteria was associated with an increased risk of developing an exacerbation.

KEYWORDS: Antibiotics, bacterial colonisation, chronic obstructive pulmonary disease, moxifloxacin

B ronchial bacterial colonisation is frequent in patients with chronic obstructive pulmonary disease (COPD) [1-3]. Studies performed using the protected specimen brush have demonstrated that up to $45 \%$ of patients with moderate-to-severe COPD carry potentially pathogenic microorganisms (PPMs) in significant concentrations in their lower airways [4]. The clinical significance of bacterial colonisation of the airways has recently been recognised. Colonised patients have increased lower airway [5-7] and systemic inflammation [8], present more frequent and severe exacerbations [9] and the changes in bacterial load and species are associated with a steeper decline in lung function over time [10]. Furthermore, one study has observed that colonised patients had more severely impaired health-related quality of life than patients whose airways were sterile [8].

Understanding the role of bacterial colonisation in COPD is crucial for the development of strategies aimed at eliminating the PPMs from the lower airways [11]. The new, more active antibiotics may allow short courses of treatment to be administered with the objective of eradicating bacteria in the airways. In this respect, moxifloxacin is a fourth generation fluoroquinolone that has demonstrated bactericidal activity against the most frequently isolated PPMs in respiratory secretions in patients with COPD [12, 13] and clinical efficacy in the treatment of exacerbations of COPD [14, 15]. Therefore, moxifloxacin is indicated in most guidelines for the treatment of exacerbations of COPD in patients with moderate-to-severe disease or with risk factors for failure [16]. The aim of the current study was to investigate the efficacy of moxifloxacin in eradication of PPMs in stable patients with COPD and the persistence of eradication over a period of 8 weeks after treatment.

\section{METHODS}

\section{Patients}

Patients $\geqslant 40 \mathrm{yrs}$ of age with COPD were recruited between January 2006 and April 2007 in two centres in the Barcelona Metropolitan Area
AFFILIATIONS

*Fundació Clínic, Institut

D'Investigacions Biomèdiques August Pi i Sunyer (IDIBAPS),

\#Dept of Pneumology, Hospital Germans Trias i Pujol, Autonomous University of Barcelona,

"Dept of Pneumology, Hospital Germans Trias i Pujol, Ciber de Enfermedades Respiratorias (CIBERES),

+Medical Dept, Bayer Schering

Pharma, and

${ }^{\S}$ Dept of Pneumology, Institut Clínic del Tòrax (IDIBAPS), Hospital Clínic, Ciber de Enfermedades Respiratorias (CIBERES), Universitat de Barcelona, Barcelona, Spain.

CORRESPONDENCE

M. Miravitlles

Servei de Pneumologia

Hospital Clínic

Villarroel 170

08036 Barcelona

Spain

E-mail: marcm@clinic.ub.es

Received:

Dec 242008

Accepted after revision:

April 022009

First published online:

April 222009 
in Spain. Inclusion criteria were as follows: smoking history of $\geqslant 10$ pack-yrs, post-bronchodilator forced expiratory volume in $1 \mathrm{~s}$ (FEV1) <60\% predicted; an increase of FEV1 with the use of $400 \mu \mathrm{g}$ of salbutamol of $<10 \%$ pred for that patient; and a ratio of post-bronchodilator FEV1 to forced vital capacity $\leqslant 0.70$ [17]. A positive sputum culture for PPMs at a concentration of $\geqslant 10^{2}$ colony-forming units $(\mathrm{CFU}) \cdot \mathrm{mL}^{-1}$ at the screening visit was required for inclusion. According to CABELLO et al. [18], PPMs were defined as those microorganisms recognised as agents causing respiratory infections, regardless of whether they belonged to the gastrointestinal or oropharyngeal flora: Gram-negative rods, such as Enterobacteriaceae and Haemophilus spp.; Gram-positive cocci, such as Staphylococcus aureus, Streptococcus pneumoniae; and Gram-negative cocci, such as Moraxella catarrhalis. Exclusion criteria were: diagnosis of asthma; any respiratory disorder other than COPD; exacerbation or use of parenteral or oral steroids or change in COPD medication in the 4 weeks before randomisation; and a course of antibiotics or hospitalisation in the 16 weeks before randomisation. Other exclusion criteria were: colonisation by Pseudomonas aeruginosa or any contraindication for the use of fluoroquinolones.

All patients gave written informed consent and the study protocol was approved by the Spanish Agency of Medicinal Products (Agencia Española del Medicamento y Productos Sanitarios; number 04-0112). Institutional review boards of both participating centres also approved the study and it was conducted according to the good clinical practice guidelines and the last version of the Declaration of Helsinki.

\section{Study design}

This was a randomised, double-blind, parallel-group, placebocontrolled trial. Patients fulfilling inclusion and exclusion criteria were screened for bronchial colonisation by PPMs in sputum. Those with sputum samples negative for PPMs were rescheduled for up to three consecutive visits at 2-week intervals to obtain a positive sputum culture. Patients with a positive sample were randomised 1:1 to receive moxifloxacin (Actira ${ }^{\circledR}$; Bayer AG, Leverkusen, Germany) 400 mg or placebo, once daily for 5 days. Active medication and matching placebo were provided by Bayer Pharma (Barcelona, Spain) and were stored and distributed by the Pharmacy Department of the Hospital Clínic (Barcelona, Spain), which also developed the randomisation tables for the study. Follow-up visits were scheduled 2 and 8 weeks after randomisation, when clinical assessment and microbiology of sputum were investigated.

Episodes of exacerbations, defined as acute episodes of increased dyspnoea, sputum production and/or purulence treated with antibiotics and/or oral corticosteroids [19], and appearing during up to 5 months of follow-up, were recorded.

\section{Measurements}

The primary efficacy end-point was the rate of eradication at visit two, 2 weeks after randomisation. Secondary end-points were rate of eradication and frequency of acquisition of new PPMs at visit three (8 weeks after randomisation), and frequency of exacerbations at 5 months of follow-up.

At randomisation, sociodemographic data, cardiovascular comorbidity, smoking habits, respiratory symptoms, and number of previous exacerbations and hospitalisations were collected. The microbiological characteristics of the sputum were recorded and all subjects performed forced spirometry and reversibility tests in the morning with the same dry rolling-seal spirometer (Spirometrics, Gray, ME, USA), according to standard techniques [20].

\section{Sputum sampling and microbiological analysis}

The sputum sample was processed within $60 \mathrm{~min}$ of collection according to standard methods [21,22]. Briefly, the patient was pretreated with an inhaled $\beta_{2}$-agonist $10 \mathrm{~min}$ before the nebulisation of isotonic saline $(0.9 \%)$ followed by increasing concentrations of hypertonic saline (3\%, $4 \%$ and $5 \%$ ), for $7 \mathrm{~min}$ with each concentration. After every induction, the patient attempted to obtain a sputum sample by coughing, and the nebulisation procedure was discontinued when the sputum volume collected was $\geqslant 1 \mathrm{~mL}$ [23]. In current smokers, sputum induction was performed after $\geqslant 6 \mathrm{~h}$ of tobacco abstinence. Sputum appearance was determined according to the MurrayWashington (MW) criteria [24] and samples with $>25$ leucocytes per field $(M W \geqslant 3)$ were considered indicative of a neutrophilic inflammatory response [25]. The sample was weighed and processed with a four-fold volume of dithiothreitol (Sputasol; Oxoid Ltd, Basingstoke, UK) and cultured. The determination of microbial typology and load was carried out by culture in selective media and serial dilutions, according to standard methods [26, 27]. For the purpose of this study, only cultures growing PPMs, such as Haemophilus influenzae, Haemophilus parainfluenzae, M. catarrhalis, S. pneumoniae, enterobacteria and/or S. aureus were considered positive, according to previously defined criteria [18, 28, 29]. Quantitative cultures were expressed as $\mathrm{CFU} \cdot \mathrm{mL}^{-1}$.

\section{Detection of microorganism DNA by PCR}

Supernatant was recovered after sputum centrifugation at $500 \times g$ for $10 \mathrm{~min}$ and stored at $-70^{\circ} \mathrm{C}$ for PCR analyses. For each PPM recovered, six colonies were stored at $-70^{\circ} \mathrm{C}$ for posterior molecular typing. DNA extraction was performed from sputum samples with negative cultures at the 2- or 8week visit in order to investigate the presence of PPM DNA using a QIAamp DNA mini kit (Quiagen, Hilden, Germany). Briefly, two pairs of specific oligonucleotide primers for the p6 gene of $H$. influenzae and $H$. parainfluenzae were designed using Primer Express software (Applied Biosystems, Foster City, CA, USA) and synthesised (Roche Diagnostics, Indianapolis, IN, USA). Since the p6 gene sequences of the $H$. influenzae and $H$. parainfluenzae differ in 30 of $274 \mathrm{pb}$, it is possible to design specific primers for each species. Oligonucleotide sequences were forward primer 5'-AATTTCCAGCTTGGTCTCCA-3' and reverse 5'-CAAAAGTTTGAGCAGCACCA-3' for $H$. influenzae, forward primer 5'-CCGTTTACTTCGGTTTCGAC-3' and reverse 5'- CAGCACGACGTTGACCTAAA-3' for $H$. parainfluenzae, and detection, forward primer 5'-GTGAGTGCCGCTTTTATAACC-3' and reverse 5'-TGTATCGCCTGCCAAGACAA-3' for $M$. catarrhalis, as previously described by GREINER et al [30]. A total of $10 \mu \mathrm{L}$ of the PCR product underwent electrophoresis in $2 \%$ agarose in Tris/borate/ EDTA buffer and visualised by ethidium bromide staining. The sizes of the amplified products, $167 \mathrm{bp}, 171 \mathrm{bp}$ and $71 \mathrm{bp}$, for $H$. influenzae, $H$. parainfluenzae and $M$. catarrhalis respectively, were compared with those of a positive control 
amplified product and a molecular mass marker ladder (Promega, Madison, WI, USA). Samples showing PPM DNA with a posterior positive culture for the same PPM in the following 3 months were considered as colonised by the PPM in spite of the negative sputum culture.

\section{Molecular typing of PPMs}

Molecular PPM typing was performed by pulse field electrophoresis to determine whether a PPM recovered from consecutive samples corresponded to the persistence of the same strain or to the acquisition of a new one [31]. Briefly, chromosomal DNA from multiple (>6) PPMs was extracted in agarose plugs. After enzyme digestion (New England Biolabs, Ipswich, MA, USA), restriction fragments were separated using a contour-clamped homogeneous electric field system (CHEF DR II; BioRad, Ivey sur Seine, France) at $14^{\circ} \mathrm{C}$ and $5 \mathrm{~V} \cdot \mathrm{cm}^{-1}$, beginning with an initial 5.6-s pulse which was linearly increased until $40.6 \mathrm{~s}$ and maintained for $25 \mathrm{~h}$ in $0.5 \mathrm{x}$ Tris/borate/EDTA (Sigma Chemical, St Louis, MO, USA). Bacteriophage lambda concatemer (New England Biolabs, Ipswich, MA, USA) was included as the molecular weight DNA marker. The patterns obtained were analysed using the Diversity Database Software (BioRad). PPMs cultured from follow-up sputum samples showing the same molecular profile by pulse field electrophoresis as those previously recovered were considered as persistent strains, whereas PPMs cultured at follow-up, which were not present in the previous sample, were considered as acquired strains.

\section{Statistical analysis}

All data were analysed using the SPSS statistical software package version 15 (Chicago, IL, USA). Results were expressed as absolute and relative frequencies for categorical variables and for continuous variables as mean $\pm \mathrm{SD}$, or median (interquartile range) when they did not follow a normal distribution. Sputum characteristics at 2 and 8 weeks after randomisation were compared between the two groups for the frequencies of PPM eradication, persistence and acquisition during follow-up and the effect of moxifloxacin treatment on these parameters (Chi-squared, Fisher exact, unpaired t-test and Mann-Whitney U-test, when appropriate). Finally, the frequency of exacerbations in both groups up to 5 months after randomisation was compared (Chi-squared, Fisher exact and unpaired t-test), and the determinants of exacerbation were calculated by logistic regression, expressing the results as odds ratios and $95 \%$ confidence intervals, considering moxifloxacin treatment, PPM persistence and acquisition as independent variables, and adjusting the models for age and lung function (postbronchodilator FEV1 \% pred). A p-value $\leqslant 0.05$ was reported as statistically significant.

\section{RESULTS}

A total of 119 consecutive stable COPD outpatients accepted to participate in the study and were screened for bronchial colonisation by PPMs. The mean age was $68 \pm 9.1$ yrs, 110 were males and the mean post-bronchodilator FEV1 was $46.2 \pm 14.1 \%$ pred. Using induced sputum, 45 (37.8\%) patients provided a positive sputum culture; this was positive for $P$. aeruginosa in five patients, who were excluded from the study. The remaining 40 patients with positive sputum cultures for PPMs other than $P$. aeruginosa were randomised to receive either placebo $(n=20)$ or moxifloxacin $(n=20)$. The sociodemographic, respiratory and microbiological profiles were comparable except for a higher exacerbation frequency in the previous year in patients treated with moxifloxacin (table 1).

On the visit week two after randomisation, PPM isolation from sputum became negative in $15(75 \%)$ out of 20 patients treated with moxifloxacin and in six (30\%) out of 20 who received placebo $(\mathrm{p}=0.01)$. Assuming a one-side contrast of hypothesis and accepting an $\alpha$ risk of 0.05 , the sample size of 20 subjects per group provides a power of $92 \%$ to identify as statistically significant the observed difference in eradication of $75 \%$ versus $30 \%$. In the placebo-treated group, the 14 cases with positive isolation of PPM consisted of 10 cases with positive sputum cultures and four cases of negative cultures but positive PCR testing followed by a positive sputum culture for the same microorganism (H. influenzae) at 8 weeks after randomisation. Furthermore, these four PPMs showed the same molecular typing profile throughout the study, from the baseline visit to the 8-week visit, confirming the persistence of the initial PPMs in the placebo group and the false negative results of the sputum culture at visit 2 (table 2). PCR testing in culturenegative sputum samples in moxifloxacin-treated patients at 2 weeks was negative in all cases (fig. 1).

\begin{tabular}{|c|c|c|}
\hline Characteristics & Placebo & Moxifloxacin \\
\hline Subjects $\mathrm{n}$ & 20 & 20 \\
\hline Age yrs & $69 \pm 10$ & $69 \pm 7$ \\
\hline Males & $19(95)$ & $19(95)$ \\
\hline Current smokers & $1(5)$ & $1(5)$ \\
\hline Tobacco consumption pack-yrs & $43 \pm 21$ & $49 \pm 24$ \\
\hline Chronic bronchitis & $19(95)$ & $20(100)$ \\
\hline Cardiovascular comorbidity & $8(40)$ & $6(30)$ \\
\hline $\begin{array}{l}\text { More than two exacerbations in previous } \\
\text { year }\end{array}$ & $6(30)$ & $12(60)$ \\
\hline $\begin{array}{l}\text { More than one hospitalisation in previous } \\
\text { year }\end{array}$ & $4(20)$ & $3(15)$ \\
\hline Post-bronchodilator FVC \% pred & $78 \pm 24$ & $70 \pm 18$ \\
\hline Post-bronchodilator FEV $1 \%$ pred & $53 \pm 16$ & $47 \pm 15$ \\
\hline Treatment with inhaled corticosteroids & $16(80)$ & $15(75)$ \\
\hline \multicolumn{3}{|l|}{ Sputum characteristics } \\
\hline Murray-Washington $\geqslant 3$ & $14(70 \%)$ & $14(70 \%)$ \\
\hline \multicolumn{3}{|l|}{ Haemophilus influenzae } \\
\hline Subjects & $11(55)$ & $8(40)$ \\
\hline Load $10^{6} \times \mathrm{CFU} \cdot \mathrm{mL}^{-1}$ & $30(0.5-60)$ & $30(1-50)$ \\
\hline \multicolumn{3}{|l|}{ Haemophilus parainfluenzae } \\
\hline Subjects & $6(30)$ & $11(55)$ \\
\hline Load $10^{6} \times \mathrm{CFU} \cdot \mathrm{mL}^{-1}$ & $0.7(0.4-188)$ & $0.6(0.1-4.2)$ \\
\hline Moraxella catarrhalis & $2(10)$ & $2(10)$ \\
\hline Streptococcus pneumoniae & $1(5)$ & $2(10)$ \\
\hline Enterobacteriaceae & $1(5)$ & 0 \\
\hline Polymicrobial & $1(5)$ & $3(15)$ \\
\hline
\end{tabular}

Data presented as mean \pm SD, $n$ (\%) or median (interquartile range), unless otherwise stated. FVC: forced vital capacity; FEV1: forced expiratory volume in $1 \mathrm{~s}$; CFU: colony forming units. 


\begin{tabular}{|c|c|c|c|}
\hline & Placebo & Moxifloxacin & $\mathrm{p}$-value \\
\hline Subjects $n$ & 20 & 20 & \\
\hline \multicolumn{4}{|l|}{ At 2-week follow-up } \\
\hline PPMs in sputum & $14(70)$ & $5(25)$ & 0.01 \\
\hline Positive sputum culture & $10(50)$ & $5(25)$ & 0.19 \\
\hline Positive PCR for PPM & $4(20)$ & 0 & 0.09 \\
\hline PPM persistence & $7(35)$ & $3(15)$ & $>0.25$ \\
\hline Acquisition of new PPM & $7(35)$ & $2(10)$ & 0.13 \\
\hline \multicolumn{4}{|l|}{ At 8-week follow-up } \\
\hline Positive sputum culture ${ }^{\#}$ & $16(80)$ & $15(75)$ & $>0.25$ \\
\hline PPM persistence & $5(25)$ & $1(5)$ & 0.18 \\
\hline Acquisition of new PPM & $12(60)$ & $14(70)$ & $>0.25$ \\
\hline \multicolumn{4}{|l|}{ PPMs at 8 weeks follow-up } \\
\hline Haemophilus influenzae & $10(50)$ & $4(20)$ & 0.10 \\
\hline Haemophilus parainfluenzae & $7(35)$ & $9(45)$ & $>0.25$ \\
\hline Pseudomonas aeruginosa & 0 & $1(5)$ & $>0.25$ \\
\hline Moraxella catarrhalis & $1(5)$ & $1(5)$ & $>0.25$ \\
\hline Polymicrobial & $2(10)$ & 0 & $>0.25$ \\
\hline \multicolumn{4}{|l|}{ At 5-month follow-up } \\
\hline Patients with exacerbation & $5(25)$ & $6(30)$ & $>0.25$ \\
\hline Time to first exacerbation days & $60 \pm 32$ & $74 \pm 39$ & $>0.25$ \\
\hline
\end{tabular}

Data are presented as $n(\%)$ or mean $\pm S D$, unless otherwise stated. PPM: potentially pathogenic micro-organism. ${ }^{\#}$ : one patient in placebo group with two different strains of $H$. influenzae, one persistent strain and one acquired at 8 weeks.

At 8 weeks after randomisation, bacterial persistence was still higher, although not significantly, in patients who received placebo than in moxifloxacin-treated patients (five (25\%) out of 20 versus one $(5 \%)$ out of 20 , respectively; $\mathrm{p}=0.18)$. However, the frequencies of acquisition of a new PPM were high and similar in both treatment groups at 8 weeks, (placebo $60 \%$ versus moxifloxacin $70 \%$; $>0.25$ ) (table 2). Due to the high incidence of acquisition of a new PPM during the 8 weeks after randomisation, the prevalence of colonisation at this time point was similar between the two treatment arms $(80 \%$ in the placebo and $75 \%$ in the moxifloxacin arm; $p>0.25$ ) (fig. 1).

The number of patients presenting an exacerbation during the 5 -month follow-up was similar in the placebo (six (30\%) out of 20 ) and the moxifloxacin groups (five (25\%) out of $20 ; \mathrm{p}>0.25$ ). The time to the first exacerbation did not significantly differ between treatment arms ( $74 \pm 39$ days versus $60 \pm 39$ days for placebo and moxifloxacin arms, respectively; $\mathrm{p}>0.25)$.

On adjusting for age and post-bronchodilator FEV1 (\% pred) in the logistic regression model, neither moxifloxacin treatment nor the presence of persistent PPM in the follow-up sputum samples was significantly associated with the appearance of an exacerbation. However, the acquisition of a new PPM during follow-up showed a statistically significant relationship with having an exacerbation (OR 9.63, 95\% CI 1.01-91.64) (table 3).

\section{DISCUSSION}

Our results have shown that a 5-day treatment course with moxifloxacin is effective in eradicating colonising bacteria in

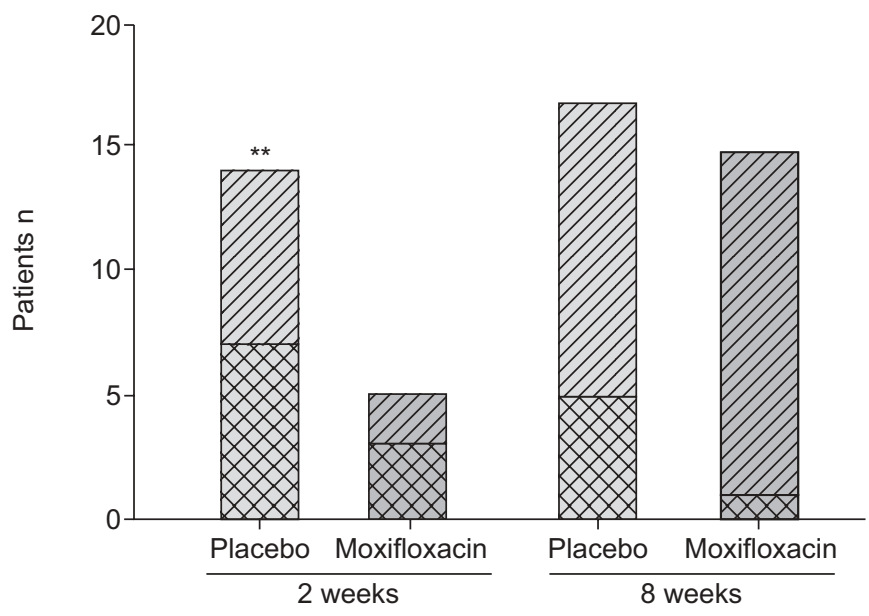

FIGURE 1. Number of patients with positive isolation of potentially pathogenic microorganisms (PPMs) in sputum at 2 and 8 weeks after randomisation. Numbers are the result of adding the percentage of patients with persistent ( $\nabla$ ) and newly acquired $(\mathbb{Z})$ strains identified by sputum culture and detection of the DNA of PPMs by PCR (table 2). **: $p=0.01$.

the airways of COPD patients. However, the eradication of bacteria is followed by the acquisition of a new colonising strain over a period of 8 weeks, in most cases, both in antibiotic-treated and untreated patients. The acquisition of a new strain is associated with an increased risk of developing an exacerbation.

Trials of antibiotic treatment of stable chronic bronchitis were conducted in the 1950s and 1960s, with inconclusive results [32] and, therefore, the prescription of prophylactic antibiotics for COPD has not been recommended. However, the chronic use of macrolides in COPD has received increasing attention in recent years. The main objective to the existing studies was to prevent exacerbations, either bacterial or viral, via mechanisms other than antibacterial activity [33-35]. In fact, two of these studies, of 3-month [34] and 1-yr duration [35], respectively, did not observe any changes in bronchial bacterial flora. However, the latter study, using erythromycin $250 \mathrm{mg}$ twice daily for 1-yr, observed a significant reduction of $35 \%$ in the frequency of exacerbations compared with placebo [35]. Instead, the rationale for the use of the fluoroquinolone moxifloxacin for the treatment of stable COPD is based on its antibacterial efficacy. Bacterial eradication reduces the inflammatory burden of the

\begin{tabular}{ll} 
TABLE 3 & $\begin{array}{l}\text { Results of the logistic regression model for the } \\
\text { variables associated with the presence of } \\
\text { exacerbation during follow-up, adjusted for age } \\
\text { and post-bronchodilator per cent predicted } \\
\text { forced expiratory volume in } 1 \mathrm{~S}\end{array}$ \\
& OR (95\% Cl) \\
\hline Variables & $0.45(0.04-4.51)$ \\
\hline $\begin{array}{l}\text { Persistence of PPM isolated at randomisation } \\
\text { Treatment with moxifloxacin } \\
\text { Acquisition of a new PPM during follow-up }\end{array}$ & $1.38(0.33-5.76)$ \\
\hline
\end{tabular}

PPM: potentially pathogenic micro-organism. 
airways [6, 36] and may eventually prevent the development of exacerbations [11, 14, 37, 38]. Treatment with moxifloxacin has been efficacious in eradicating bacteria from the airways but has neither prevented the occurrence of exacerbations nor prolonged the time to the first exacerbation in our population. It is important to consider that our study, although adequately powered for the main end-point, was clearly underpowered to demonstrate an effect in reduction of exacerbations or prolonging exacerbation-free interval. A previous randomised trial comparing moxifloxacin with amoxycillin, clarithromycin or cefuroxime in the treatment of exacerbations of COPD observed a significantly prolonged time free from exacerbation in favour of moxifloxacin (133 days versus 118 days; $p=0.03$ ) associated with a significantly better eradication rate for the quinolone $[12,14]$. Interestingly, to achieve sufficient statistical power, the study included a total of 730 patients followed for 9 months after the exacerbation [14]. In contrast, an uncontrolled study in the late 1980s in patients colonised with $H$. influenzae observed persistence of 11 out of 14 strains of the microorganism after treatment with ampicillin, doxycycline, erythromycin or tetracycline, but the strains did not become resistant to the antimicrobial administered; instead, the low penetration of these antibiotics into the sputum was most likely to be responsible for the poor outcome [39].

Our results suggest that the sterilising effect of moxifloxacin in the airways vanishes after $\sim 2$ months. Therefore, to maintain the bronchial tree as free from bacteria as possible, either continuous or recurrent courses of treatment would be necessary. In this case, special attention must be paid to the possible development of resistance to the fluoroquinolone by the colonising bacteria. However, our results suggest that only exceptionally persistent strains would be repeatedly exposed to the antibiotic over time with an increased risk of development of resistance. Most of the time, newly acquired strains are exposed to the antibiotic and effectively cleared from the airways without the possibility of becoming resistant to the antibiotic. However, the long-term use of the antibiotic in this indication should be restricted to the controlled clinical trial setting, and the efficacy, safety and potential development of resistance carefully assessed.

The use of PCR and molecular typing has allowed the identification of four false-negatives for colonisation at 2 weeks among patients in the placebo group. These were very likely to be patients with a low or very low bacterial load in the airways, with sputum cultures that were negative by the usual microbiological techniques. Similarly, using the same methodology, MuRPHY et al. [40] demonstrated that episodes of negative sputum cultures preceded and followed by identical strains of $H$. influenzae were, in fact, periods of continuous colonisation by the same strain of $H$. influenzae identified by detection of strain-specific DNA in some of the sputum samples that yielded negative cultures.

A major limitation of our study is the small sample size, which did not allow verification of the hypothesis that eradication of colonising bacteria results in a prolonged time to the first exacerbation; additionally, the number of subjects with frequent exacerbations was higher in the moxifloxacin group. It is of note that this treatment is not indicated for patients colonised with $P$. aeruginosa. Nonetheless, this colonisation occurs only in a minority of patients with COPD (only $4.2 \%$ in our screened population), and many of these episodes are transient colonisation [41]. The inclusion of $H$. parainfluenzae in the group of PPMs may be controversial; however, this microorganism was included in the original definition of PPMs [18] and has been considered a respiratory pathogen, together with $H$. influenzae, in many studies dealing with bacterial colonisation in COPD $[6,7,9,18,28]$. In fact, MidDLETON et al. [42] demonstrated that $H$. parainfluenzae can be a pathogen in the lower respiratory tract when impaired airway defences delay bacterial clearance, as is the case in COPD. In any case, the frequency of isolation of this microorganism, both at inclusion and at 8 weeks, was similar in both treatment groups; therefore, excluding this isolation from the analysis would have not modified the results.

Of note, the study has several strengths, the randomised, double-blind design, the use of molecular typing to detect colonisation and re-colonisation by the same or different strain and, particularly, the inclusion of patients with demonstrated bacterial colonisation at baseline. Even if large, randomised, controlled trials ever demonstrate the efficacy of this therapeutic approach in reducing exacerbations and/or lung damage in COPD, this treatment will be restricted to selected subgroups of patients due to the risk of development of resistance and potential side-effects. The patients with demonstrated bacterial colonisation with sensitive bacteria are an obvious target population for this prophylactic treatment and the current study has demonstrated the efficacy of a short course of moxifloxacin in bacterial eradication in this context. Large clinical trials are required to answer some of the questions which remain open regarding antibiotic treatment of bacterial colonisation in COPD.

\section{SUPPORT STATEMENT}

This study was funded by Bayer Schering Pharma (Berlin, Germany) and a grant from La Marató de TV3 (Barcelona, Spain).

\section{STATEMENT OF INTEREST}

Statements of interest for M. Miravitlles, E. Monsó, C. de la Roza, J. Morera and A. Torres, and for the study itself, can be found at www. erj.ersjournals.com $/ \mathrm{misc} /$ statements.dtl

\section{ACKNOWLEDGEMENTS}

The authors would like to thank D. Soy (Pharmacy Dept, Hospital Clínc, Barcelona, Spain) for the handling of the double-blind medication and the randomisation of the patients.

\section{REFERENCES}

1 Monsó E, Ruiz J, Rosell A, et al. Bacterial infection in chronic obstructive pulmonary disease. A study of stable and exacerbated outpatients using the protected specimen brush. Am J Respir Crit Care Med 1995; 152: 1316-1320.

2 Zalacain R, Sobradillo V, Amilibia J, et al. Predisposing factors to bacterial colonisation in chronic obstructive pulmonary disease. Eur Respir J 1999; 13: 343-348.

3 Monsó E, Rosell A, Bonet G, et al. Risk factors for lower airway bacterial colonisation in chronic bronchitis. Eur Respir J 1999; 13: 338-342.

4 Weinreich UM, Korsgaard J. Bacterial colonisation of lower airways in health and chronic lung disease. Clin Respir J 2008; 2: 116-122. 
5 Soler N, Ewig S, Torres A, et al. Airway inflammation and bronchial microbial patterns in patients with stable chronic obstructive pulmonary disease. Eur Respir J 1999; 14: 1015-1022.

6 Hill AT, Campbell EJ, Hill SL, et al. Association between airway bacterial load and markers of airway inflammation in patients with stable chronic bronchitis. Am J Med 2000; 109: 288-295.

7 Sethi S, Maloney J, Grove L, et al. Airway inflammation and bronchial bacterial colonisation in chronic obstructive pulmonary disease. Am J Respir Crit Care Med 2006; 173: 991-998.

8 Banerjee D, Khair OA, Honeybourne D. Impact of sputum bacteria on airway inflammation and health status in clinical stable COPD. Eur Respir J 2004; 23: 685-691.

9 Patel IS, Seemungal TAR, Wilks M, et al. Relationship between bacterial colonisation and the frequency, character, and severity of COPD exacerbations. Thorax 2002; 57: 759-764.

10 Wilkinson TMA, Patel IS, Wilks M, et al. Airway bacterial load and FEV1 decline in patients with chronic obstructive pulmonary disease. Am J Respir Crit Care Med 2003; 167: 1090-1095.

11 Miravitlles M. Exacerbations of chronic obstructive pulmonary disease: when are bacteria important? Eur Respir J 2002; 20: Suppl. $36,9 \mathrm{~s}-19 \mathrm{~s}$

12 Niederman MS, Anzueto A, Sethi S, et al. Eradication of H. influenzae in AECB: a pooled analysis of moxifloxacin phase III trials compared with macrolide agents. Respir Med 2006; 100: 1781-1790.

13 Miravitlles M. Moxifloxacin in the management of exacerbations of chronic bronchitis and COPD. Int J COPD 2007; 2: 191-204.

14 Wilson R, Allegra L, Huchon G, et al. Short-term and long-term outcomes of moxifloxacin compared to standard antibiotic treatment in acute exacerbations of chronic bronchitis. Chest 2004; 125 953-964.

15 Miravitlles M, Molina J, Brosa M. Clinical efficacy of moxifloxacin in the treatment of exacerbations of chronic bronchitis: a systematic review and meta-analysis. Arch Bronconeumol 2007; 43 $16-21$.

16 Miravitlles M, Monsó E, Mensa J, et al. Antimicrobial treatment of exacerbation in chronic obstructive pulmonary disease: 2007 consensus statement. Arch Bronconeumol 2008; 44: 100-108.

17 Rabe KF, Hurd S, Anzueto A, et al. Global strategy for the diagnosis, management, and prevention of chronic obstructive pulmonary disease: GOLD executive summary. Am J Respir Crit Care Med 2007; 176: 532-555.

18 Cabello H, Torres A, Celis R, et al. Bacterial colonisation of distal airways in healthy subjects and chronic lung disease: a bronchoscopic study. Eur Respir J 1997; 10: 1137-1144.

19 Cazzola M, MacNee W, Martinez FJ, et al. Outcomes for COPD pharmacological trials: from lung function to biomarkers. Eur Respir J 2008; 31: 416-468.

20 American Thoracic Society. Standardization of spirometry: 1987 Update. Am Rev Respir Dis 1987; 136: 1285-1298.

21 Pin I, Gibson PG, Kolendowicz R, et al. Use induced sputum cell counts to investigate airway inflammation in asthma. Thorax 1992; 47: 25-29.

22 Pizzichini E, Pizzichini MM, Efthimiadis A, et al. Indices of airway inflammation in induced sputum: reproducibility and validity of cell and fluid-phase measurements. Am J Respir Crit Care Med 1996; 154: 308-317.

23 Aaron SD, Angel JB, Lunau M, et al. Granulocyte inflammatory markers and airway infection during acute exacerbation of chronic obstructive pulmonary disease. Am J Respir Crit Care Med 2001 163: 349-355.

24 Murray PR, Washington JA. Microscopic and bacteriologic analysis of expectorated sputum. Mayo Clin Proc 1975; 50: 339-344.

25 Van Scoy RE. Bacterial sputum cultures. A clinician's viewpoint Mayo Clin Proc 1977; 52: 39-41.

26 Balows A, Hausler WJ, Herrmann KL, et al., Manual of Clinical Microbiology. 5th Edn. Washington, American Society of Microbiology, 1991.

27 Pye A, Stockley RA, Hill SL. Simple method for quantifying viable bacterial numbers in sputum. J Clin Pathol 1995; 48: 719-724.

28 Sethi S, Sethi R, Eschberger K, et al. Airway bacterial concentrations and exacerbations of chronic obstructive pulmonary disease. Am J Respir Crit Care Med 2007; 176: 356-361.

29 Murphy TF, Brauer AL, Sethi S, et al. Haemophilus haemolyticus: a human respiratory tract commensal to be distinguished from Haemophilus influenzae. J Infect Dis 2007; 195: 81-89.

30 Greiner O, Day PJ, Altwegg M, et al. Quantitative detection of Moraxella catarrhalis in nasopharyngeal secretions by real-time PCR. J Clin Microbiol 2003; 41: 1386-1390.

31 Yano H, Suetake M, Kuga A, et al. Pulsed-field gel electrophoresis analysis of nasopharyngeal flora in children attending a day care center. J Clin Microbiol 1999; 38: 625-629.

32 Black P, Staykova T, Chacko E, et al. Prophylactic antibiotic therapy for chronic bronchitis. Cochrane Database Syst Rev 2003; 1: CD004105.

33 Suzuki T, Yanai M, Yamaya M, et al. Erythromycin and common cold in COPD. Chest 2001; 120: 730-733.

34 Banerjee D, Khair OA, Honeybourne D. The effect of oral clarithromycin on health status and sputum bacteriology in stable COPD. Respir Med 2005; 99: 208-215.

35 Seemungal TAR, Wilkinson TMA, Hurst JR, et al. Long-term erythromycin therapy is associated with decreased chronic obstructive pulmonary disease exacerbations. Am J Respir Crit Care Med 2008; 178: 1139-1147.

36 White AJ, Gompertz S, Bayley DL, et al. Resolution of bronchial inflammation is related to bacterial eradication following treatment of exacerbations of chronic bronchitis. Thorax 2003; 58: 680-685.

37 Rosell A, Monso E, Soler N, et al. Microbiologic determinants of exacerbation in chronic obstructive pulmonary disease. Arch Intern Med 2005; 165: 891-897.

38 Chodosh S. Clinical significance of the infection-free interval in the management of acute bacterial exacerbations of chronic bronchitis. Chest 2005; 127: 2231-2236.

39 Groeneveld K, van Alphen L, Eijk PP, et al. Endogenous and exogenous reinfection by Haemophilus influenzae in patients with chronic obstructive pulmonary disease: the effect of antibiotic treatment on persistence. J Infect Dis 1990; 161: 512-517.

40 Murphy TF, Brauer AL, Schiffmacher AT, et al. Persistent colonisation by Haemophilus influenzae in chronic obstructive pulmonary disease. Am J Respir Crit Care Med 2004; 170: 266-272.

41 Murphy TF, Brauer AL, Eschberger K, et al. Pseudomonas aeruginosa in chronic obstructive pulmonary disease. Am J Respir Crit Care Med 2008; 177: 853-860.

42 Middleton AM, Dowling RB, Mitchell JL, et al. Haemophilus parainfluenzae infection of respiratory mucosa. Respir Med 2003, 97: 375-381. 